\title{
071 IS TRAVEL COST A SIGNIFICANT DETERRENT TO THE HEALTH-SEEKING BEHAVIOR OF HOUSEHOLD- BENEFICIARIES UNDER CONDITIONAL CASH TRANSFERS (CCT) AND/OR PHILHEALTH INSURANCE'S SPONSORED PROGRAM?
}

Mel Lorenzo Mabazza Accad. University of the Philippines, School of Economics, Quezon City, Philippines

\subsection{6/bmjopen-2015-forum2015abstracts.71}

Background Six out of ten Filipinos die without seeing a health professional (UP NHI, 2010). Some of the leading causes of mortality are preventable with early detection and diagnosis. Actual cost of medical care is not the sole reason for not seeking medical care as even indigent members who are entitled to free medical insurance have very low utilization rate of health care benefits (NDHS).

These make the facilitation of the meeting of patients and healthcare providers more important. A wide array of reasons for low utilization of health care exists. For instance, in a 2013 study by Faraon, et.al, high transaction costs affect a portion of those who underutilized health insurance benefits. Lack of accessible supply-side factors such as roads and effective mode of transportation are partly to be blamed for in these transaction costs.

Objectives This paper contributes to existing literature as it verifies the effect of travel costs on the households' decision to seek medical care after controlling for many government social protection programs, ie. Conditional Cash Transfer (CCT) and Social Health Insurance for Indigent Program.

Methods This paper uses the Millennium Challenge Corporation's 2012 baseline randomized control trial (RCT) data designed for the impact evaluation of KALAHI-CIDSS Program (2nd phase). The follow up survey is not yet available but was already conducted last first quarter of 2014 .

After controlling for many other variables, this study will test the effects of travel costs to each of following dependent variables, (1) expenditure for medical care, (2) decision to consult with a healthcare provider.To solve the possible endogeneity of these costs even after controlling for other observed variables, the costs were instrumented, then regressed on the response of households to existence of serious illness in the family (sought help, etc) to compute the Local Average Treatment Effect (LATE). To further test the technique-robustness of the estimate, this study computed the Average Treatment Effect on the Treated (ATT) using Propensity Score Matching (PSM) methods.If the baseline samples' follow up survey (done last first quarter in 2014) will be available before the deadline, Average Treatment Effects (ATE) will be computed by Difference-in-Difference using Fixed Effects method.

Result Initial results from the baseline RCT data suggest a negative relationship between travel costs and the decision to seek medical treatment from list of households" options.

Conclusion The conclusions support existing literatures that point to the importance of infrastructures to enhance peoples" access to health care. Intuitively, CCT beneficiaries regard the effect of travel costs less than PhilHealth insurance, as the latter virtually expands households" budget. 